\title{
Differences between Male and Female College Students in Dynamic Stability Change through Measuring Lower Extremity Muscle Fatigue
}

\author{
Hae sung Lee ${ }^{1 *}$, Sung-Sik Ko ${ }^{1 *}$, Keun-Ok An ${ }^{1 * *}$ \\ ${ }^{1}$ Korea National University of Transportation, Chungju, Korea \\ * These two authors contributed equally to this work
}

Received: June 12, 2019

Accepted: July 09, 2019

Published online: July 30, 2019

Keywords:

Dynamic Stability

Gender Difference

Muscle Fatigue

Y Balance Test

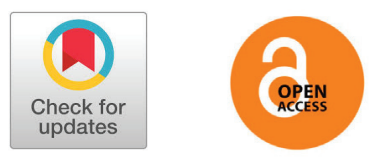

\section{ABSTRACT}

OBJECTIVES The purpose of this study was to compare and analyze differences by gender, changes in dynamic stability due to lower extremity muscle fatigue.

METHODS A total of 47 college-age students participated in the experiment. Subjects consisted of 29 males and 19 females. A muscle fatigue squat protocol with Smith machine and $Y$-balance Test was conducted and measurements were taken before and after the onset of fatigue.

RESULTS There were statistically significant differences before and after fatigue in anterior right leg $(p<.001)$, anterior left leg $(p<.001)$, posteromedial right leg $(p=.011)$, posteromedial left leg $(p=.010)$, pesterolateral left leg $(p=.014)$, total score right leg $(p<.001)$ and total score left leg $(p<.001)$, the only exception being posterolateral right leg $(p=.057)$. The difference between male and female groups decreased in a statistically significant manner on posteromedial left leg $(p=.045)$ and posterolateral right leg $(p=.031)$. The effects of interaction between measurement periods did not represent statistically significant differences in all categories.

CONCLUSIONS The result of this study represented a decrease in total score of $Y$-balance Test before and after the application of muscle fatigue. There were no gender differences in the dynamic stability of male and female subjects due to muscle fatigue.

๑ The Asian Society of Kinesiology and the Korean Academy of Kinesiology
서론

스포츠 상황에서 근육의 피로와 손상은 매우 밀접한 관계이며 잠재적인 부상 위험 요인으로 분류된다[1]. 대표적인 하지 부상으로 분류되는 무릎의 앞십자인대 (anterior cruciate ligament) 손상의 경우 비접촉성 손 상의 비율이 $70 \%$ 이상을 차지하고 있으며[2], 하지 근 피로를 유발하는 훈련을 하거나 피로의 누적이 가중되 는 시합 후반에 주로 발생한다[3]. 이는 축구선수의 비

**Correspondence: Keun-Ok An, Korea National University of Transportation 50 Daehak-ro, Chungju-si, Chungbuk, Republic of Korea.; Tel: +82-043-841-5995; Email: koan@ut.ac.kr
접촉성 손상에 관한 연구에서도 확인할 수 있는데, 부상 률이 시합 종료 약 15 분 전 혹은 시합 후반부에 프로축 구선수와 유소년 축구선수 모두 부상 빈도가 급격히 높 아지는 경향을 보인다[4]. 따라서 하지의 근피로가 근 신경 기능과 균형능력에 부정적인 영향을 미쳐 전반적 인 안정성의 저하를 초래한다[3].

동적 안정성은 움직이는 상황에서 발생하는 외부로 부터의 자극에 대해 균형을 잃지 않고 신체의 움직임을 제어할 수 있는 전반적인 능력을 뜻한다. 동적 안정성 을 평가하는 Y balance test는 하지의 동적 안정성 검 사인 $\mathrm{SEBT}$ (star excursion balance test)의 절차를 간 
소화하고 검사의 반복성을 높이고 하지의 근력과 고유 수용성 감각을 측정할 수 있는 평가로 현장에서 활용이 매우 용이하다[5]. 또한 Y balance test는 동적 안정성 을 평가할 뿐만 아니라 부상 위험을 예측하는 지표로써 사용되기도 하는데, 남자 미식축구 선수를 대상으로 $\mathrm{Y}$ balance test를 실시한 결과 하지 길이를 세 방향 도달 거리의 합으로 나눈 뒤 정규화 한 값이 $89 \%$ 이하로 나 타난 경우, 부상의 위험이 유의하게 증가한다[6].

근피로는 고강도 유형의 운동이나 장시간 동안 실 시하는 운동으로 발생하는 대사성 피로 물질의 축적에 의해 신경 전달 속도가 감소되어 근육의 수축력이 감소 하고 이로 인해 힘을 유지하지 못하는 현상을 의미한다 [7]. 하지 근육의 피로 누적은 구심성 혹은 원심성으로 이루어지는 신경 전달의 속도를 감소시켜 [3] 근신경 조 절 능력 혹은 관절의 위치감각 인지 능력에 영향을 준다 [8]. 위치감각 인지 능력의 저하는 운동 수행력의 감소 와 직접적인 연관이 있다고 알려져 있고[9], 선행 연구 에 의하면 근피로가 위치감각 인지 능력의 저하와 연관 이 있음을 확인할 수 있다[10]. 하지 근육의 피로는 무 릎 주변부 근육의 운동조절 변화, 반응 시간의 변화 그 리고 근신경의 변화를 유발해 부상에 노출될 수 있으며 [11] 관절 각도의 신전에도 영향을 미쳐 외부의 충격 에 의해 관절이 쉽게 손상을 입을 수 있다는 연구 결과 [12]와 근피로에 의해 발생한 균형 능력의 저하가 하지 손상의 요인이 될 수 있다는 연구 결과[3]를 보았을 때, 하지의 근피로와 동적 안정성 사이에는 밀접한 연관이 있음을 알 수 있다.

근육의 피로 수준이 정적인 상황에서의 균형 유지능 력이나 동적인 상황에서의 안정상태를 유지할 수 있는 능력에 미치는 영향을 규명하기 위해 실제 운동 상황과 비슷한 유형의 피로를 줄 수 있는 기능적 근피로 프로토 콜을 적용한 이후 눈감고 외발 서기 혹은 사이드 커팅 동 작과 같은 변인들을 이용한 선행 연구들이 있다 $[12,13]$.

선행 연구를 살펴보면 하지 근육에 피로를 적용한 집 단에서 한발 서기 자세에서의 안정성이 크게 떨어지거 나[14] 피로가 사이드 커팅 동작 수행 시 하지 관절의 각도와 움직임이나[12] 동적 자세 조절 능력에 전반적 으로 영향을 미쳤다고 하였다[13]. 그러나 피로 요인이 결과에 큰 영향을 미친 요인이라고 보기에는 무리가 있 거나[12] 피로가 무릎의 동적 안정성에 직접적인 영향 을 미치는가에 대해 상관관계가 명확하지 않다는 연구
결과[15]들을 살펴보면 피로가 동작 수행에는 영향을 주지만 동적 안정성이나 전반적인 균형 능력의 저하와 직접적인 연관성이 있는가에 대한 의문점은 아직 명확 하게 해결되지 못한 부분이 있다.

또한 성별에 따른 무릎 앞십자인대 비접촉성 손상은 남성보다는 여성에게서 더 높은 비율로 손상이 꾸준히 발생하고 있다[16]. 여성이 남성보다 손상에 더 취약한 요인으로는 근신경 조절 능력의 차이[17], 근력 및 근 지구력의 차이 $[18,19]$ 등이 언급된다. 이러한 선행 연 구에서 나타난 성별에 따른 손상비율의 차이로 근피로 에 따른 동적 안정성의 변화 역시 성별에 따라 차이가 있을 수 있음을 시사한다.

하지 근피로에 따른 동적 안정성의 변화를 살펴보고 그것을 통해 손상 위험을 예측하기 위해 근피로 프로토 콜을 적용한 이후 Y balance test를 실시하여 연구한 사례는 부족한 실정이다. 만일 근피로가 동적 안정성 검 사인 Y balance test의 결과에 영향을 준다면 그 결과 를 이용하여 부상 위험의 예측 및 성별에 따른 차이도 고려할 수 있을 것이며 운동 대상자에 대한 동적 안정 성 검사를 통해 위험군을 선별함으로써 근피로로 인한 운동 손상 예방에 기여할 수 있을 것이다. 따라서 이 연 구는 현장에서 활용이 용이한 Y balance test를 이용하 여 하지 근육의 피로에 따른 남녀 간의 차이에 대해 비 교 분석하고자 한다.

\section{연구방법}

\section{1. 연구 대상}

실험의 참가자는 $\mathrm{K}$ 대학교 체육 학과에 재학 중인 학 생들로 하였다. 실험 시작에 앞서 하지의 근골격계에 기 능적으로 문제가 있는 학생들을 선별하여 실험에서 제 외하기 위해 진단된 근골격계 질병의 유무에 관한 조사 를 하였으며 기능적인 문제가 없는 참가자들 중 참여 의 사를 분명히 밝힌 학생들을 대상으로 동의서 작성 및 실 험의 목적에 대한 설명과 참여 시 주의사항에 대해 고 지하였다. 피험자의 신체적 특성은 <Table 1 >과 같다.

\section{2. 연구설계}

실험을 위해 피험자에게 실험당일 일상생활 수준의 신체 활동을 제외한 근육에 피로를 줄 수 있는 활동들 을 피하게 하였고 정확한 근육의 피로 상태를 만들기 위 해 에너지 드링크나 기타 보충제 및 카페인 음료의 실험 
Table 1. Physical characteristics of subjects

\begin{tabular}{lllllll}
\hline Gender & Age(yrs) & Height(cm) & $\begin{array}{l}\text { R Leg } \\
\text { Length(cm) }\end{array}$ & $\begin{array}{l}\text { L Leg } \\
\text { Length(cm) }\end{array}$ & BMI(kg/m²) & Bodyfat(\%) \\
\hline Male $(\mathrm{n}=29)$ & $19.00 \pm 0.99$ & $177.30 \pm 5.16$ & $90.40 \pm 4.30$ & $90.50 \pm 4.27$ & $22.35 \pm 2.24$ & $16.62 \pm 2.71$ \\
Female $(\mathrm{n}=18)$ & $19.28 \pm 1.27$ & $162.62 \pm 5.28$ & $81.42 \pm 3.53$ & $81.58 \pm 3.49$ & $21.36 \pm 2.06$ & $26.67 \pm 3.49$ \\
\hline $\begin{array}{l}\text { R; Right, L; Left, BMI; Body Mass Index } \\
\text { Values are expressed as M } \pm \text { SD }\end{array}$ & & & & & & \\
\hline
\end{tabular}

전 섭취를 금지하도록 교육하였다. 실험 시작 30 분 전 부터 정확한 스쿼트 동작에 대한 교육을 실시하였으며, 실험 중 위험한 상황에 대비하여 측정자를 도와줄 보조 자를 배치하였다.

\section{3. 측정방법}

\section{(1) Y balance test}

Y balance test는 피험자가 시험대 위에 서서 측 정하는 발을 중앙의 발판을 지지하고 반대쪽 발로 각 각 $120^{\circ}$ 간격으로 전방(anterior; ANT), 후방 내측 (posterior medial; PM), 후방 외측(posterior lateral; $\mathrm{PL}$ ) 방향으로 뻗어 있는 선을 따라 균형을 잃지 않는 선 에서 발판을 최대한 멀리 밀어내어 거리를 측정하는 방 법이다[20]. 피험자가 정확한 동작으로 실험을 진행할 수 있고 연습으로 인한 학습효과를 방지하기 위해 사전 연습은 4 6회로 제한하여 실시하였으며, 지지하는 발 혹은 내딛는 발 쪽의 균형이 무너져 자세를 유지하지 못 하거나 지지하는 발의 뒤꿈치가 들리고 내딛는 발을 밀 어내야 하는 발판 위에 얹어 놓아 균형을 잡는 경우 혹 은 내딛는 발끝으로 발판을 차서 밀어내는 경우 실패로 간주하고 검사를 다시 실시하였다.

테스트 순서는 우측 전방, 좌측 전방, 우측 후내 측, 좌측 후내측, 우측 후외측, 좌측 후외측 순으로 실시하였고, 모든 검사는 각각 두 번씩 실시하였으며 두 번의 검사 중 더 높은 값을 측정값으로 채택하였다.

측정값의 정규화를 위해 피험자의 하지 길이를 위앞 엉덩뼈가시의 가장 아랫부분부터 안쪽 복사뼈의 가장 원 위부까지 부드러운 줄자로 센티미터 단위로 측정하였다.

측정값의 총점(total score)은 우측, 좌측 각각 3 방 향(전방, 후내측, 후외측) 뻗은 거리의 합을 '하지길이 X3'으로 나눈 다음 100 을 곱하였으며, 각 3방향(전방, 후내측, 후외측)의 점수는 각각 뻗은 거리를 하지길이
로 나눈 다음 100을 곱하여 정규화(normalization)하 였다<Table 2>.

\begin{tabular}{ll}
\multicolumn{2}{l}{ Table 2. Score normalization formula } \\
\hline Score & Formula \\
\hline Total score & $\begin{array}{l}\text { (anterior+ posterior medial+ posterior lateral)/ } \\
(3 \times \text { leg length }) \times 100 \\
\text { (reach distance/leg length }) \times 100\end{array}$ \\
\hline
\end{tabular}

\section{(2) 근피로 프로토콜}

하지 동적 안정성 검사를 종료한 이후 실시한 근피 로 프로토콜은 선행 연구에서 이용한 근피로 프로토콜 $[12,21]$ 을 기초로 하여 연구의 방향에 맞게 수정, 보완 하여 이용하였다. 근피로 프로토콜은 스미스 머신(Smith machine)을 이용하여 여성은 $30 \mathrm{~kg}$ 의 중량을 남성은 $50 \mathrm{~kg}$ 의 중량으로 스쿼트를 실시하였고, 개인별 차이를 고려하여 스쿼트를 5회 1세트를 기준으로 최대 15세트 반복 수행을 목표로 더 이상 수행할 수 없을 때까지 지 속적으로 실시하였다[12].

피험자는 근피로 프로토콜이 종료된 직후 하지의 근피로가 회복되기 전에 휴식 없이 곧바로 Y balance test를 실시하였고 실험이 진행되는 도중에 발생할 수 있는 피험자의 근피로 회복을 최소화하기 위해 세트마다 맨몸 스쿼트를 10회씩 수행하도록 하였다[11].

\section{자료처리}

본 연구의 가설 검증을 위하여 각 집단의 종속 변인 별 평균 및 표준편차를 산출하였다. 또한 측정 시기(피로 전, 피로 후)와 집단(남자, 여자)에 따른 종속 변인들의 차이는 반복 측정 이원변량분석(two-way repeatedmeasures ANOVA)으로 분석하였다. 이 연구의 가설 검 증을 위해서 SPSS 20.0 통계 프로그램을 사용하였고, 통계적 유의 수준은 $\mathrm{p}<.05$ 로 설정하였다. 


\section{결과}

근피로 적용 전후에 따른 반복 측정 이원변량분석 결 과를 살펴보면 <Table 3>과 같다.

\begin{tabular}{|c|c|c|c|c|}
\hline & & YBT(\%) & PF-YBT(\%) & $\mathbf{p}$ \\
\hline \multirow{2}{*}{$\begin{array}{l}\text { ANT } \\
\text { R }\end{array}$} & Male & $68.82 \pm 9.08$ & $65.25 \pm 9.46$ & \multirow{2}{*}{$\begin{array}{l}A:<.001 * * \\
B: .641 \\
A^{*} B: .095\end{array}$} \\
\hline & Female & $71.46 \pm 7.27$ & $65.00 \pm 9.38$ & \\
\hline \multirow{2}{*}{$\begin{array}{l}\text { ANT } \\
\text { L }\end{array}$} & Male & $67.69 \pm 9.04$ & $65.33 \pm 8.45$ & \multirow{2}{*}{$\begin{array}{l}A:<.001 * * \\
B: .641 \\
A^{*} B: .095\end{array}$} \\
\hline & Female & $72.84 \pm 8.86$ & $67.89 \pm 9.95$ & \\
\hline \multirow{2}{*}{$\begin{array}{l}\mathrm{PM} \\
\mathrm{R}\end{array}$} & Male & $119.33 \pm 10.22$ & $118.14 \pm 9.02$ & \multirow{2}{*}{$\begin{array}{l}A: .011^{*} \\
B: .065 \\
A^{*} B: .215\end{array}$} \\
\hline & Female & $125.17 \pm 6.42$ & $121.86 \pm 8.24$ & \\
\hline \multirow{2}{*}{$\begin{array}{l}\mathrm{PM} \\
\mathrm{L}\end{array}$} & Male & $118.60 \pm 8.44$ & $117.42 \pm 9.02$ & \multirow{2}{*}{$\begin{array}{l}A: .010^{* *} \\
B: .045^{*} \\
A^{*} B: .163\end{array}$} \\
\hline & Female & $124.63 \pm 5.16$ & $120.84 \pm 8.93$ & \\
\hline \multirow{2}{*}{$\begin{array}{l}\mathrm{PL} \\
\mathrm{R}\end{array}$} & Male & $116.08 \pm 10.13$ & $115.63 \pm 8.56$ & \multirow{2}{*}{$\begin{array}{l}A: .057 \\
B: .031^{*} \\
A^{*} B: .123\end{array}$} \\
\hline & Female & $123.12 \pm 7.26$ & $118.98 \pm 7.71$ & \\
\hline \multirow{2}{*}{$\begin{array}{l}\mathrm{PL} \\
\mathrm{L}\end{array}$} & Male & $118.13 \pm 11.22$ & $115.53 \pm 8.87$ & \multirow{2}{*}{$\begin{array}{l}A: .014^{*} \\
B: .110 \\
A^{*} B: .945\end{array}$} \\
\hline & Female & $122.23 \pm 5.22$ & $119.48 \pm 7.63$ & \\
\hline \multirow{2}{*}{$\begin{array}{l}\text { Total } \\
\text { R }\end{array}$} & Male & $101.41 \pm 8.11$ & $99.68 \pm 7.26$ & \multirow{2}{*}{$\begin{array}{l}A:<.001^{* * *} \\
B: .080 \\
A^{*} B: .054\end{array}$} \\
\hline & Female & $106.59 \pm 5.85$ & $101.93 \pm 7.49$ & \\
\hline \multirow{2}{*}{$\begin{array}{l}\text { Total } \\
\mathrm{L}\end{array}$} & Male & $101.51 \pm 8.08$ & $99.42 \pm 7.08$ & \multirow{2}{*}{$\begin{array}{l}A:<.001 \\
B: .052 \\
A^{*} B: .184\end{array}$} \\
\hline & Female & $106.56 \pm 5.49$ & $102.74 \pm 7.92$ & \\
\hline \multicolumn{5}{|c|}{$\begin{array}{l}{ }^{*} \mathrm{p}<.05{ }^{* *} \mathrm{p}<.01{ }^{* * *} \mathrm{p}<.001 \\
\mathrm{YBT} ; \mathrm{Y} \text { Balance Test, PF YBT; Post Fatigue Y Balance Test, ANT; Anterior, } \\
\mathrm{PM} \text {; Posterior Medial, PL; Posterior Lateral, R; Right, L; Left } \\
\mathrm{A} \text {; measurement period (before and after fatigue), B; group (gender), } \\
\mathrm{A}^{*} \mathrm{~B} ; \text { interaction between } \mathrm{A} \text { and } \mathrm{B} \\
\text { Values are expressed as M } \pm \mathrm{SD}\end{array}$} \\
\hline
\end{tabular}

측정 시기와 집단간의 상호작용 효과는 없는 것으로 나타났다. 측정 시기(피로 전, 피로 후)에 따라 오른쪽 하지의 후외측( $\mathrm{p}=.057)$ 을 제외한 모든 방향에서 통계 적으로 유의한 수준에서 감소하였다. 집단(남자, 여자) 간의 차이는 왼쪽 하지의 후내측 $(\mathrm{p}=.045)$ 과 오른쪽 하 지의 후외측 $(\mathrm{p}=.031)$ 을 제외한 모든 방향에서 통계적 으로 유의한 결과를 나타내지는 않았다.

\section{논의}

이 연구는 성별에 따른 하지 근피로에 대한 동적 안 정성의 변화를 알아보기 위해 남성과 여성에게 각각 근
피로 프로토콜을 이용하여 하지에 근피로를 적용하기 전 과 후의 Y balance test의 변화를 분석하였다.

결과를 전반적으로 살펴보게 되면 피로 전후에 대 한 결과는 근피로가 동적 자세 조절 능력 및 균형 능력 에 전반적인 영향을 미쳤다고 밝힌 선행 연구 결과와 일치하였다 $[12,13,14]$. Y balance test에서 총점 (total score)의 저하는 근골격계 손상과 밀접한 연관이 있다고 밝힌 선행 연구[6]와 비교하여 볼 때, 근피로 적용 전보다 적용 후에 값이 통계적으로 유의한 수준 으로 감소하는 것은 근피로가 하지 손상의 원인이 될 수 있음을 시사하고 있으며, 이러한 결과는 다수의 선 행연구에서 근피로가 하지 손상의 주요인이라고 밝힌 선행 연구들의 의견과도 일치한다[3,11]. 따라서, 근 피로와 동적 안정성은 밀접한 연관이 있으며 근피로 수준과 부상의 위험은 밀접한 관련이 있다는 것을 재 확인하였다.

근피로 전후 총점(total score)를 분석해 보면, 집 단(남자, 여자) 간에 통계적으로 유의한 차이를 발견하 지 못했고 시기와 집단 간의 상호작용 효과 역시 없는 것으로 나타났다. 이것은 여성이 근피로에 대한 내성이 높다고 발표한 선행 연구 $[22,23]$ 나 성별 간 근신경 조 절 능력 혹은 근력, 근지구력의 차이 $[17,18,19]$ 로 여 성이 근신경 반응 능력의 변화에 더욱 민감하다[15]는 선행 연구들의 의견과는 일치하지 않은 결과이다.

또한 근육의 피로 뿐만 아니라, 하지를 지지하고 있 는 표면의 상태가 안정적인 상황보다는 불안정한 표면 에서 동적 안정성에 부정적인 영향을 미쳤다는 연구결 과로 볼 때, 근육의 피로와 마찬가지로 안정적이지 않 은 표면 또한 동적안정성에 영향을 미치는 것을 알 수 있다[24]. 따라서 근피로 적용의 유형, 동적안정성에 영향을 주는 요인 및 동적 안정성 평가 방법 등 좀 더 다양한 방법으로 남성과 여성의 성별 차이에 대한 후 속연구가 필요할 것으로 판단된다.

위 결과들을 종합해보면 근피로에 적용 이후 동적 안정성을 측정한 결과 남성과 여성 모두 부정적인 영 향을 받은 것을 확인할 수 있었고, 남성과 여성 모두 근피로에 의해 동일한 수준의 영향을 받았으며 성별에 따른 차이는 나타나지 않았다는 것을 이 연구를 통해 알 수 있었다.

\section{결론 및 제언}

근피로 적용 전후 $\mathrm{Y}$ balance test의 총점(total 
score)에 감소가 나타나 근피로에 따라 남녀 모두에 게 동적 안정성 감소에 영향을 주었으나, 근피로에 따른 동 적 안정성 감소의 남녀간에 성차는 없는 것으로 나타났 다. 또한 근피로 적용의 유형, 동적안정성에 영향을 주 는 요인 및 동적 안정성 평가 방법 등 좀 더 다양한 방법 으로 남성과 여성의 성별 차이에 대한 후속연구가 필요 할 것으로 판단된다.

\section{Conflicts of Interest}

The authors declare no conflict of interest.

\section{References}

1. Kraemer WJ, Gardiner DF, Gordon SE, et al. Differential effects of exhaustive cycle ergometry on concentric and eccentric torque production. J Sci Med Sport, 2001; 4(3):301-309.

2. Boden BP, Dean GS, Feagin JA, Garrett WE. Mechanisms of anterior cruciate ligament injury. Orthopedics, 2000; 23(6):573-578.

3. Yuk JY, Hwang HJ, Kwon MG. Influence of leg muscle fatigue on the balance ability, Korea Sport Res; 17(5):643-647

4. Hawkins RD, Fuller CW. A prospective epidemiological study of injuries in four English professional football clubs. Br J Sports Med, 1999; 33(3):196-203

5. Plisky PJ, Gorman PP, Butler RJ, Kiesel KB, Underwood FB, Elkins B. The reliability of an instrumented device for measuring components of the star excursion balance test. N Am J Sports Phys Ther, 2009; 4(2):92.

6. BUTLER RJ, Lehr ME, Fink ML, Kiesel KB, Plisky PJ. Dynamic balance performance and noncontact lower extremity injury in college football players: an initial study. Sports Health, 2013, 5.5: 417-422.

7. Edwards RH. Biochemical bases of fatigue in exercise performance: catastrophe theory of muscular fatigue. Biochemistry of exercise, 1983; 13:3-28.

8. Gribble PA, Hertel J, Eenegar CR, Buckley WE. The effects of fatigue and chronic ankle instability on dynamic postural control. J Athl Train, 2004; 39(4):321.
9. Barrack RL, Skinner HB, Buckley SL. Proprioception in the anterior cruciate deficient knee. Am J Sports Med, 1989; 17(1):1-6.

10. Marks R. Effect of exercise-induced fatigue on position sense of the knee. Aust J Physiother, 1994; 40(3):175181.

11. James CR, Scheuermann BW, Smith MP. Effects of two neuromuscular fatigue protocols on landing performance. J Electromyogr Kinesiol, 2010; 20(4):667675.

12. Park EJ, Lee YS, Lim BO, Kim YW, Lee KK. Effect of Anticipation and Fatigue on Lower Extremity Joint Loads during Side-cutting in Female Soccer Player. Korean J Sports Med, 2011; 29(1):26-36.

13. Kim SGN, Lee SC, Kim CY, Jeong HS, Lee SY. Effect of functional fatigue protocol on static and dynamic postural control of lower extremities. Korean J Sport Sci, 2018; 29(4):603-612

14. Lim GL, Chang JS. The effects of muscle fatigue of knee muscles on balance ability in healthy adults. J Korean Med Rehabil, 2018; 28(2):105-111.

15. Wojtys EM, Huston LJ, Schock, HJ, Boylan, J. P, AshtonMiller, JA. Gender differences in muscular protection of the knee in torsion in size-matched athletes. J Bone Joint surg Am, 2003; 85:782-789.

16. Prodromos, CC, Han Y, Joyce B, Shi KA, Meta-analysis of the anterior cruciate ligament tears as a function of gender, sport, and a knee injury-reduction regimen. Arthroscopy, 2010; 23(12):1320-1325

17. Hewett TE, Paterno MV, Myer GD. Strategies for enhancing proprioception and neuromuscular control of the knee. Clin Orthop Relat Res. 2002; 402:76-94.

18. Huston LJ, Wojtys EM. Neuromuscular performance characteristics in elite female athletes. Am J Sports Med, 1996; 24(4):427-436.

19. Wojtys EM, Huston LJ. Neuromuscular performance in normal and anterior cruciate ligament-deficient lower extremities. Am J Sports Med, 1994; 22(1):89-104.

20. Shaffer SW, Teyhen DS, Lorenson CL, Lorenson CL, Warren PL, Koreerat CM, Straseske CA, Childs JD. Y-balance test: a reliability study involving multiple 
raters. Mil Med, 2013; 178(11):1264-1270

21. Gehring D, Melnyk M, Gollhofer A. Gender and fatigue have influence on knee joint control strategies during landing. Clin Biomech, 2009; 24(1):82-87.

22. Mannion AF, Dolan P. Electromyographic median frequency change during isometric contraction of the back extensors to fatigue. Spine. 1994; 19(11):12231229.
23. Umezu Y, Kawazu T, Tajima F, Ogata H. Spectral electromyographic fatigue analysis of back muscles in healthy adult women compared with men. Arch Phys Med Rehabil. 1998; 79(5):536-8.

24. Reghabi S, Power L, An KO, Ko J. Comparison between the Modified Star Excursion Balance Test and the Traditional Star Excursion Balance Test in Recreational Athletes. Asian J Kinesiol. 2018; 20(3):19-23. 